\title{
Extraction Method of Harmonic Signal from Chaotic Background Based on Effective Data Segment \\ Peili CONG
}

Information Engineering Department, Liaoning Mechatronics College, Liaoning Dandong, 118009, China

Congpeili@yeah.net

\section{Keywords: Chaotic Signal; Harmonic Signal; EMD; Effective Data Segment}

\begin{abstract}
To further improve the performance of extraction of harmonic signal from chaotic background, an effective data segment based separation method for single channel signal was proposed. The single channel signal is mixed by chaotic signal and harmonic signal, and empirical mode decomposition (EMD) can transform the single channel signal into positive definite separation problem by intrinsic mode function (IMF). However, EMD has the endpoint effect problem which results in the signal distortion on boundary. Hereby we use the effective data segment of IMF to carry on the independent component analysis (ICA) processing to obtain the separation matrix, which can avoid the distortion signal effect on the result. Computer simulation verified that the proposed method is high availability.
\end{abstract}

\section{Introduction}

Chaotic signal has been paid much attention and some achievements have been arrived at. Chaos is a common phenomenon in various fields such as secure communications [1], electronic warfare and ECG, EEG signal processing etc. Especially in secure communication, chaotic communication provides an effective way for the security of the transmission information [2]. However, extracting useful information from chaotic background is a difficult problem in chaotic communication [3]. For the single channel mixed chaotic signal, the signal often needs to be decomposed into some components, which can transform the problem to positive definite separation. The decomposition methods include EMD, local mean decomposition (LMD) and wavelet transform etc. In many decomposition methods, EMD is adaptive and widely used in engineering. However, EMD has the endpoint effect problem, which leads to the distortion of the boundary signal. Furthermore, the distortion signal will spread to the internal signal, which affects the accuracy of some data of IMF. To further improve the performance of extraction of harmonic signal from chaotic background by EMD and ICA, an effective data segment based separation method for single channel signal was proposed. The mixed signal of harmonic signal and chaotic signal is decomposed by EMD to obtain IMF components, and the instantaneous frequency of the IMF is used to determine the starting and ending time of the effective data segment. The separation matrix is solved by the IMF components of the effective data segment rather than the whole IMF components. After obtaining the separation matrix, the original mixed signal is separated by the separation matrix, and the harmonic signal can be extracted. The proposed method can effectively avoid the effect of EMD endpoint effect on the extraction of harmonic signals. At last, computer simulation was done to verify that the proposed method is high availability.

\section{The Model of Signal Mixing and Separation}

Blind source separation (BSS) comes from a cocktail party for speech signal separation, which is applied to the separation of the mixture of independent signals. If the delay of signal transmission is ignored, the general mixed problem can be expressed by the instantaneous mixing model. According to the relationship between the number of received signals and the source signal, the BSS problem can be divided into positive definite separation, underdetermined separation and 
overdetermined separation, among which the underdetermined separation it refer to the number of the received signal is less than the source signal. The underdetermined separation problem is a more difficult and widespread problem in BSS. The typical methods to solve the problem of the underdetermined separation are sparse representation and positive definite transformation, in which positive definite transformation method is simple and easy to understand. Single channel signal separation is a typical underdetermined separation problem. Fig. 1 shows the model of the signal and separation diagram.

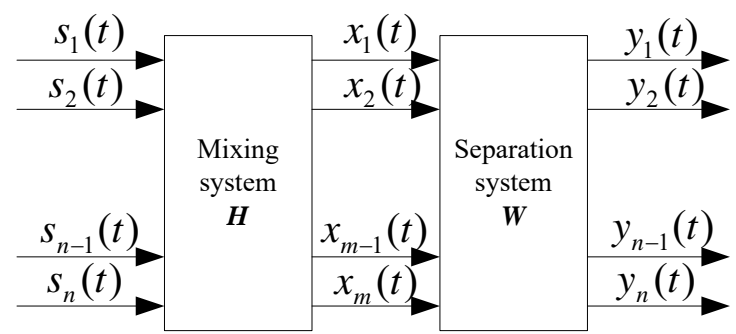

Fig.1 The model of signal mixing and separation

where $S=\left[s_{1}(t), s_{2}(t), \mathrm{L}, s_{n}(t)\right]^{\mathrm{T}}$ is the unknown and zero mean independent source signals, $\boldsymbol{X}=\left[x_{1}(t), x_{2}(t), \mathrm{L}, x_{m}(t)\right]^{\mathrm{T}}$ is the $m$-dimension zero mean random observation vector and $\boldsymbol{Y}=\left[y_{1}(t), y_{2}(t), \mathrm{L}, y_{n}(t)\right]^{\mathrm{T}}$ is the signals after blind separation. Let the mixing system $\boldsymbol{H}$ be full rank matrix of $m \times m$, the linear mixed process can be given by

$$
\boldsymbol{X}=\boldsymbol{H} \boldsymbol{S}=\sum_{j=1}^{m} h_{j} S_{j}
$$

where $\boldsymbol{H}$ denotes the unknown channel mixing matrix which can be shown as follow

$$
\boldsymbol{H}=\left[\begin{array}{cccc}
h_{11} & h_{12} & \mathrm{~L} & h_{1 m} \\
h_{21} & h_{22} & \mathrm{~L} & h_{2 m} \\
\mathrm{M} & \mathrm{M} & \mathrm{L} & \mathrm{M} \\
h_{m 1} & h_{m 2} \mathrm{~L} & h_{m m}
\end{array}\right]=\left[\mathbf{h}_{1}, \mathbf{h}_{2}, \mathrm{~L} \mathbf{h}_{m}\right]
$$

The separation model can be shown as follow

$\boldsymbol{Y}=\boldsymbol{W} \times \boldsymbol{X}=\boldsymbol{W} \times \boldsymbol{H} \times \boldsymbol{S}$

When $\boldsymbol{W}=\boldsymbol{H}^{-1}$, separated $\operatorname{signal} \boldsymbol{Y}=\hat{\boldsymbol{S}}$, we get the estimated value of source signals. It's important to point that this paper only extracts harmonic signal under the chaotic backgrounds, thus, as long as the similarity of extracted harmonic signal and source signal is high enough, it is determined to reach the requirements of proposed algorithm in this paper. For the blind harmonic signal extraction under the chaotic background, the source signals consist of different frequency harmonic signals, and the chaotic ones. If the source signals can meet the definite solution conditions of ICA, ICA can solve the problem of the blind harmonic signal extraction.

\section{Single Channel Signal Separation by EMD-ICA}

EMD was proposed by N. E. Huang for nonstationary signal processing, which decomposes the signal into series of components with intrinsic physical property. The decomposition process of EMD is in essence a sifting process [4]. The purpose of EMD is to divide the signal into a series of IMF components with a narrower frequency range and as such easier for further processing. EMD uses envelope defined by local maximal and minimal values to calculate the average curve. The upper envelope is obtained by connecting all of the local maximal points found in the signal by three-order spline interpolation function. Similarly, the lower envelope is obtained by connecting all the local minimum points of the signal by three-order spline interpolation function. All data of the signal should be confined between the upper and lower envelopes. Let $m_{1}$ is the mean value of the upper and lower envelope and $h_{1}$ is the difference between the original signal and $m_{1}$, then 
$x(t)-m_{1}(t)=h_{1}(t)$

If $h_{1}(t)$ meets the condition of IMF, it is then the first IMF components of the signal $x(t)$. Otherwise, it takes $h_{1}(t)$ as a new signal to repeat above steps $k$ times until the decomposition signal $I_{1}(t)$ meets the condition of IMF.

$$
\begin{aligned}
& h_{1, k-1}(t)-m_{k}(t)=h_{1, k}(t) \\
& I_{1}(t)=h_{1, k}(t)
\end{aligned}
$$

Where $I_{1}(t)$ is the IMF component of the original signal $x(t)$ with the highest frequency. Subtracting $I_{1}(t)$ from the original signal $x(t)$ to obtain $x^{\prime}(t)$, and then it takes $x^{\prime}(t)$ as a new signal to repeat above steps to obtain the $\operatorname{IMF} I_{2}(t)$. If all the IMF components have been decomposed from the signal $x(t)$, the EMD process is finished and then $x(t)$ can be expressed as

$$
x(t)=\sum_{i=1}^{n} I_{i}(t)+r_{n}(t)
$$

where $r_{n}(t)$ is the residual component with monotone trend.

Once the single channel signal is decomposed into series IMF components, FastICA can be used to implement BSS and the harmonic signal can be extracted. The implementation process of single channel signal separation by EMD-ICA can be shown in Fig.2.

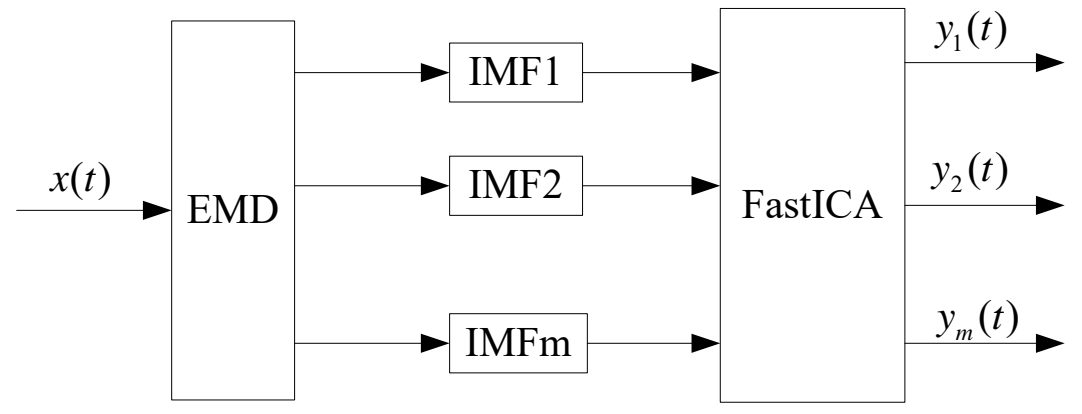

Fig.2 Implementation process of BSS by EMD-ICA for single channel signal

Where $x(t)$ is the mixing signal with chaotic signal and harmonic signal. IMF is obtained by EMD FastICA separates IMF to independent components denoted by $y_{i}(t), i=1,2, \mathrm{~L}, m$. If the separation is successful, the harmonic signal can be extracted from the chaotic background. For EMD has endpoint effect [5] and the data on the boundary will be distortion, we can select the effective data segment of IMF according to the instantaneous frequency for FastICA.

\section{Computer Simulation and Analysis}

In the simulations, the chaotic signal adopt the $x$ branch circuit of the Lorenz chaotic signal, The three-dimensional Lorenz chaotic system shows different states with different parameter values, the system would enter the chaotic state when $a=28, b=8 / 3$ and $c=10$. Lorenz chaotic signal can be given by [6]

$$
\left\{\begin{array}{c}
d x / d t=-a(x-y) \\
d y / d t=-x z+b x-y \\
d z / d t=x y-c z
\end{array}\right.
$$

The harmonic signals use sinusoidal signal with center frequency $f_{1}=5 \mathrm{~Hz}$ and $f_{2}=25 \mathrm{~Hz}$ and the sample frequency is $f_{s}=1000 \mathrm{~Hz}$. To simplify the simulation process, it assumes that the mixing signal has no noise interference. The purpose of this paper is to study the harmonic signal extraction from chaotic background, Noise suppression can be achieved through a variety of ways, so this hypothesis does not affect the simulation results of the argument. The EMD result is shown in Fig.3. The effective data segment according to instantaneous frequency of IMF is between 
$t=0.5 \mathrm{~s}$ and $t=2 \mathrm{~s}$. The separation result of FastICA is shown in Fig.4.
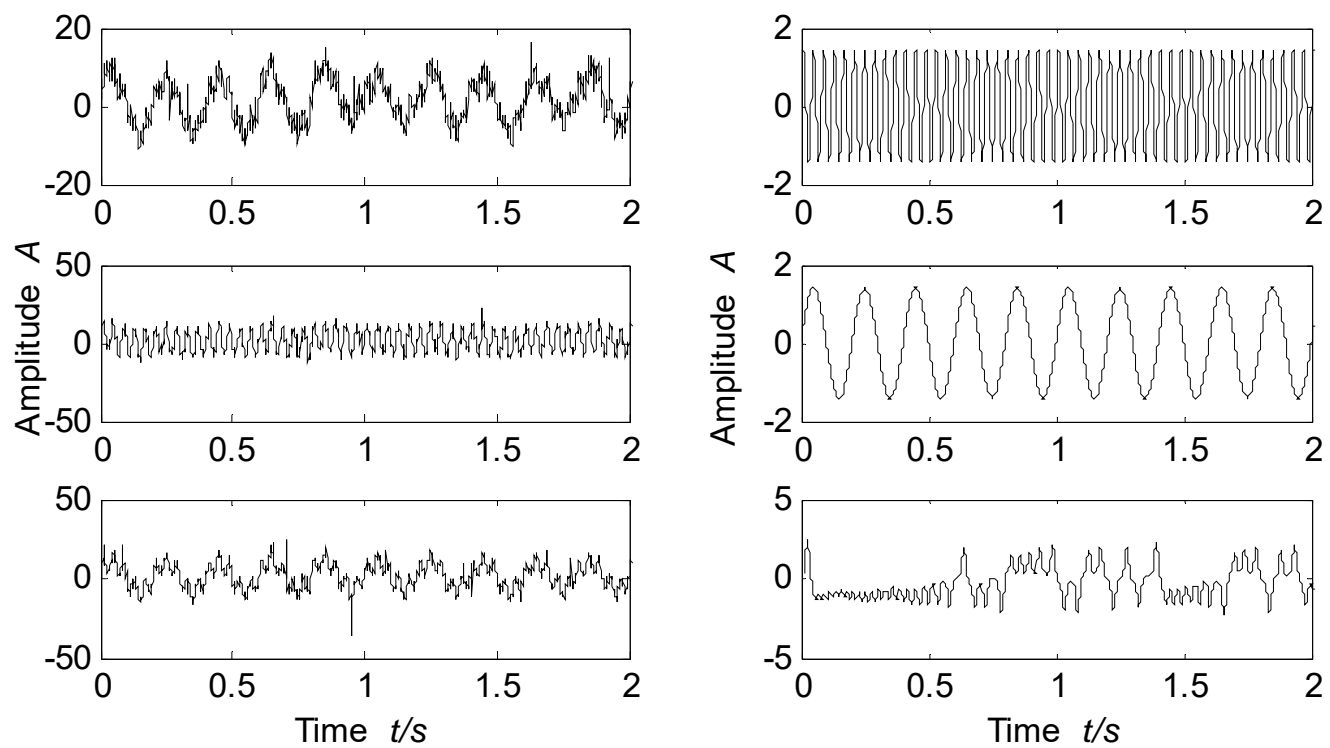

Fig.3 Result of EMD

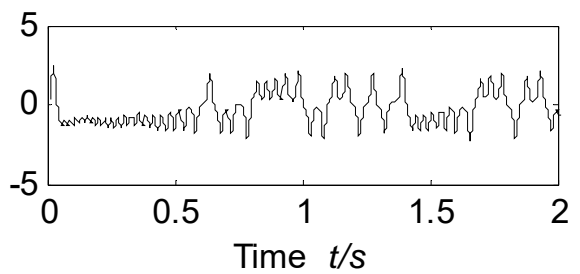

Fig.4 Result of FastICA

Fig. 4 shows the separation signals by the method proposed in this paper. Using FastICA to extract the harmonic signal from the IMF directly, the best coefficient of the similarity coefficient matrixes is only 0.875 , and the mainly coefficients in the similarity coefficient matrixes all can reach to 0.992 by the proposed method.

\section{Conclusion}

This work proposed an extraction method of harmonic signal from chaotic background based on the effective data segment, which uses EMD for the single channel mixing signal to obtain IMF, and then the effective data segment determined by the instantaneous frequency of IMF. The separation matrix only depends on the effective data segment of IMF by FastICA, which can improve the accuracy of the separation matrix. As a result, the harmonic signal extraction performance is improved. The simulation result shows the effectiveness of the proposed method.

\section{References}

[1] WANG E. F., WANG D. Q. Extraction method of harmonic signal from chaotic background based on time-frequency [J]. Journal of Engineering of Heilongjiang University, 2012, 3(1): 105-110.

[2] LI Y., YANG B. J., DENG X. Y., et al. The chaotic detecting method for the frequency of harmonic signal [J]. Journal of Electronics \& Information Technology, 2005, 27(5): 731-733.

[3] WANG E. F., WANG D. Q., DING Q. New time-frequency method of harmonic signal extraction in chaotic secure communication system [J]. Journal on Communications, 2011, 32(12): 60-64.

[4] XIAO Y., YIN F. L. Decorrelation EMD: a new method of eliminating mode mixing [J]. Journal of Vibration and Shock, 2015, 34(4): 25-29.

[5] DU C. Y., ZHANG Y. F., YANG P., et al. Approaches for the end effect restraint of empirical mode decomposition algorithm [J]. Chinese Journal of Scientific Instrument, 2009, 30(1): 55-60.

[6] A. Schwarz, K. Reindl, and W. Kellermann. A two-channel reverberation suppression scheme based on blind signal separation and wiener filtering [C]. Acoustics, Speech and Signal Processing (ICASSP), 2012 IEEE International Conference on , 113-116. 